\title{
Positive effects of meal frequency and calorie restriction on antioxidant systems in rats
}

\author{
Hasan Basri Savas, ${ }^{1}$ Fatih Gultekin, ${ }^{1}$ Ibrahim Metin Ciris ${ }^{2}$ \\ ${ }^{1}$ Department of Medical Biochemistry, Alanya Alaaddin Keykubat University Faculty of Medicine, Antalya, Turkey \\ ${ }^{2}$ Department of Pathology, Suleyman Demirel University Faculty of Medicine, Isparta, Turkey
}

\begin{abstract}
OBJECTIVE: In living organisms, there is a balance between the oxidant and antioxidant systems. Reactive products continuously formed by exogenous and endogenous sources are rendered harmless by the antioxidant system. Oxidative stress is an etiological factor in aging and the development of various diseases. In the present study, the aim was to investigate the effects of meal frequency and calorie restriction on oxidant-antioxidant systems in rat serum and tissue.
\end{abstract}

METHODS: Nine adult male Wistar Albino rats were used for the pilot study, and another 24 adult male Wistar Albino rats, also weighing 200 to $250 \mathrm{~g}$ each, were included in the main study. The rats were divided into 3 groups based on nutrition: the ad libitum group (AL) ( $n=8)$, the 2-meal group (TM) $(n=8)$, and the 2-meal with calorie restriction group (TM-CR) $(n=8)$. Following the 4-week pilot study, nutrition regulation was performed in all groups for 20 weeks, 7 days a week, with 60 minutes allotted per meal. Serum and tissues of rats were isolated at the end of the experiment. Total antioxidant status (TAS) and total oxidant status (TOS) were determined using the Erel method. Oxidative stress index (OSI) was calculated using the formula OSI $=$ TOS/TAS. Liver tissue was examined histopathologically. Statistical analyses were performed using the IBM SPSS Statistics for Windows, Version 20.0 (IBM Corp., Armonk, NY, USA) program.

RESULTS: There were significant differences between the AL and TM, and the AL and TM-CR groups in adipose tissue TOS and OSI, and between the AL and TM groups in the liver TAS of the rats $(p<0.05)$.

CONCLUSION: Calorie restriction and sparse meal frequency can increase the activity of antioxidants and can reduce oxidative stress. Thus, many diseases caused by oxidative stress may be prevented with the correct regulation of feeding.

Keywords: Antioxidant status; caloric restriction; meal frequency; nutrition; oxidant status; rat.

$\mathrm{N}$ utrition is defined as the process by which organisms take in nutritional elements from the external environment and use them to facilitate all functions of living [1]. The effect of nutrition on metabolism is a subject that has been examined by various researchers for years [2-6]. Studies investi-

A part of this study was previously presented at the " $6^{\text {th }}$ World Congress of Oxidative Stress, Calcium Signaling and TRP. 24-27 May 2016, Isparta, Turkey. (www.cmos.org.tr)." as an oral presentation entitled "The effects of meal frequency and calorie restriction on oxidant-antioxidant systems in rats."

Received: January 30, 2017 Accepted: May 05, 2017 Online: August 26, 2017

Correspondence: Dr. Hasan Basri SAVAS. Alanya Alaaddin Keykubat Universitesi Tip Fakultesi,

Tibbi Biyokimya Anabilim Dali, Antalya, Turkey.

Tel: +90 242 - 5181144 e-mail: hasan.savas@alanya.edu.tr

(c) Copyright 2017 by Istanbul Northern Anatolian Association of Public Hospitals - Available online at www.kuzeyklinikleri.com 
gating the effects of meal frequency on metabolism are now rather outdated, as they examined the effects on metabolic enzymes and hormones without investigating the antioxidant system [7-11]. It is well established that excessive energy intake in humans increases the risk of diabetes, cancer, and cardiovascular diseases. However, the effects of increased meal frequency on human health or life span are not clear [12]. Increased meal frequency in humans has been linked to hepatic steatosis, an increase in triglycerides, and obesity [13]. Calorie restriction in animals and humans is a $20 \%$ to $40 \%$ reduction in the intake of calories compared with feeding ad libitum $[14,15]$. Oxidative stress, mitochondrial damage, inflammation, and changes in the structure of proteins-DNA play an important role among the factors that induce neurodegeneration. It is thought that decreased oxidative stress and stimulated neutrophil production may be part of a mechanism related to the reduction of neurodegenerative changes observed with calorie restriction [16-19]. Calorie restriction reduces oxidative stress and the production of oxidant agents [20]. Several other studies have also found that calorie restriction reduced oxidative stress [21-23]. Dubnov et al. conducted a study in which $60 \%$ calorie restriction was implemented for 40 days. As a result of the experiment, they found that calorie restriction reduced oxidative stress in various tissues [21]. It was also observed in another study that antioxidant capacity decreased and there was a reduction of lipid peroxidation and protein oxidation products in the group with calorie restriction [24]. One of the accepted theories is that mild calorie restriction reduces the damage caused by free radicals [25]. It has been reported that antioxidant activity increased and oxidative stress decreased with calorie restriction [26]. It was demonstrated in a study of rats that calorie restriction increased antioxidant activity in the serum and liver tissue homogenate in comparison with the control group [27]. There is a continuous production of free radicals in organisms as a result of the effects of oxygen on metabolic pathways and exposure to radiation, drugs, and harmful chemicals. These free radicals facilitate oxidative stress. Antioxidant system enzymes disarm these free radicals and ensure that balance is preserved in the organism and life is sustained. Increase in oxidative stress and deficiency in the antioxidant system result in the pathogenesis of more than 100 diseases [28]. In living organisms, there is a balance between the oxidant and antioxidant systems. Reactive products continuously formed by exogenous and endogenous sources are rendered harmless by the antioxidant system [28].

In the present study, the aim was to investigate the effects of meal frequency and calorie restriction on oxidant-antioxidant systems in rat serum and adipose, brain, and striated muscle tissues, the liver, and liver pathology.

\section{MATERIALS AND METHODS}

\section{Animals}

A total of 33 male Wistar Albino rats weighing 200 to $250 \mathrm{~g}$ each were used in the experimental procedures. The ambient temperature and relative humidity of the room where the animals were housed was $21 \pm 1^{\circ} \mathrm{C}$ and $60 \pm 7 \%$, respectively. The room was illuminated with artificial light in a 12 hour dark/light cycle. The animals were allowed free access to tap water, but standard pellet food was consumed in a controlled environment. All studies were performed with the approval of the Suleyman Demirel University ethics committee for animal experiments (approval date: 21.05.2015; number: 21438139-172).

\section{Experimental design}

Before initiating the studies, all of the rats were exposed to a light/dark living cycle for a week. Darkness was provided for 12 hours overnight to encourage them to sleep at night and be active during the day. Each rat was housed in a standard Euro-type 2 small animal cage (Techniplast, S.p.A., Buguggiate, Italy).

\section{Pilot study}

The rats were divided into 3 groups based on nutrition: the ad libitum group (AL) $(n=3)$, the 2-meal group (TM) $(n=3)$, and the 2 -meal with calorie re- 
striction $(20 \%)$ group (TM-CR) ( $n=3)$. In the first week, all groups were given 20 minutes of access to food at determined feeding times. Duration of access was increased gradually to 30 minutes in the second week, 45 minutes in the third week, and an hour in the fourth week. The amount of food consumed at feedings was determined by calculating the difference in the weight of the food in the cages before and after feeding. As a result of the 4-week preliminary study, the average amount of food a rat consumed in a day was determined. The ideal amount of time for the rats to consume the determined amount of food was found to be 1 hour. It was decided to provide the daily meals of the TM and TM-CR groups from 9:00 to 10:00 every morning and from 4:00 to 5:00 every evening. In the pilot study, it was found that the rats in the $\mathrm{AL}$ group consumed $0.062 \mathrm{~g}$ of food per gram of body weight. Based on this result, when the necessary calculations were made regarding the weight of the animals in the main study, it was calculated that the TM group and the TM-CR group should consume 20 and $16 \mathrm{~g} /$ day of food, respectively.

\section{Main study}

The rats were divided into 3 groups based on nutrition: ad libitum (AL) group $(\mathrm{n}=8), 2$-meal (TM) group $(\mathrm{n}=8)$, and 2 -meal with calorie restriction (20\%) group (TM-CR) $(n=8)$. The nutrition regulation was performed in all groups for 20 weeks, 7 days a week, with 60 minutes allotted per meal following the 4-week pilot study. The AL group was provided with a sufficient amount of food (ad libitum). The TM group was fed by dividing the amount calculated as daily food intake into 2 meals. The TM-CR group was fed by dividing the amount calculated as daily food intake reduced by $20 \%$ into 2 meals. The AL, TM, and TM-CR groups were all fed using the same standard food. There were no restrictions on water in any group.

\section{Anesthesia and preparation of}

\section{blood and tissue samples}

After 20 weeks, blood samples were taken and rats were euthanized using $10 \%$ ketamine (Alfamine;
Alfasan IBV, Woerden, The Netherlands) and 2\% xylazine (Alfazine; Alfasan IBV, Woerden, The Netherlands) in anesthesia. Serum and tissue samples were isolated.

\section{Obtaining the serum}

The blood samples obtained were centrifuged at $3500 \mathrm{rpm}$ for 8 minutes (Rotanta 460; Andreas Hettich GmbH \& Co. KG, Tuttlingen, Germany) and serum was collected. Serum samples were divided into Eppendorf tubes (Eppendorf AG, Hamburg, Germany) and stored at $-800 \mathrm{C}$ until the date of analysis (Daihan WiseCryo WUF; Daihan Scientific, Wonju-si, Gangwon-do, South Korea).

\section{Tissue homogenization}

Adipose, striated muscle, and liver tissue were weighed on assay balance (Scaltec SPB33; Scaltec Instruments $\mathrm{GmbH}$, Goettingen, Germany) and homogenized by mixing with phosphate buffer at a ratio of $1 / 10$ (Ultra-Turrax T25; Janke \& Kunkel/IKA, Staufen im Breisgau, Germany and UW2070; Bandelin Electronic GmbH \& Co. KG, Berlin, Germany). The homogenates were centrifuged for 10 minutes at $+4^{\circ} \mathrm{C}$ at $10,000 \mathrm{~g}$. In the following stage, supernatants were stored at $-80^{\circ} \mathrm{C}$ in the cryogenic freezer until they were tested.

\section{Total antioxidant status, total oxidant status, and oxidative stress index analyses}

Serum and tissue samples were thawed and mixed using a vortex-mixer (Labinco L 46; Labinco BV, Breda The Netherlands) for oxidant-antioxidant analysis. The serum total antioxidant status (TAS), total oxidant status (TOS), and oxidative stress index (OSI) values were measured spectrophotometrically using modified Erel method and biochemical auto-analyzer (AU5800; Beckman Coulter, Inc., Brea, CA, USA) with TAS and TOS commercial kit (Rell Assay Diagnostics, Gaziantep, Turkey) $[29,30]$. The TAS and TOS results in the serum were expressed in $\mu \mathrm{mol}$ Trolox Eq/L and $\mu \mathrm{mol}$ $\mathrm{H}_{2} \mathrm{O}_{2}$ Eq/L, respectively. The TAS and TOS results for the tissues were expressed via division by the protein value. OSI was calculated using the formula 

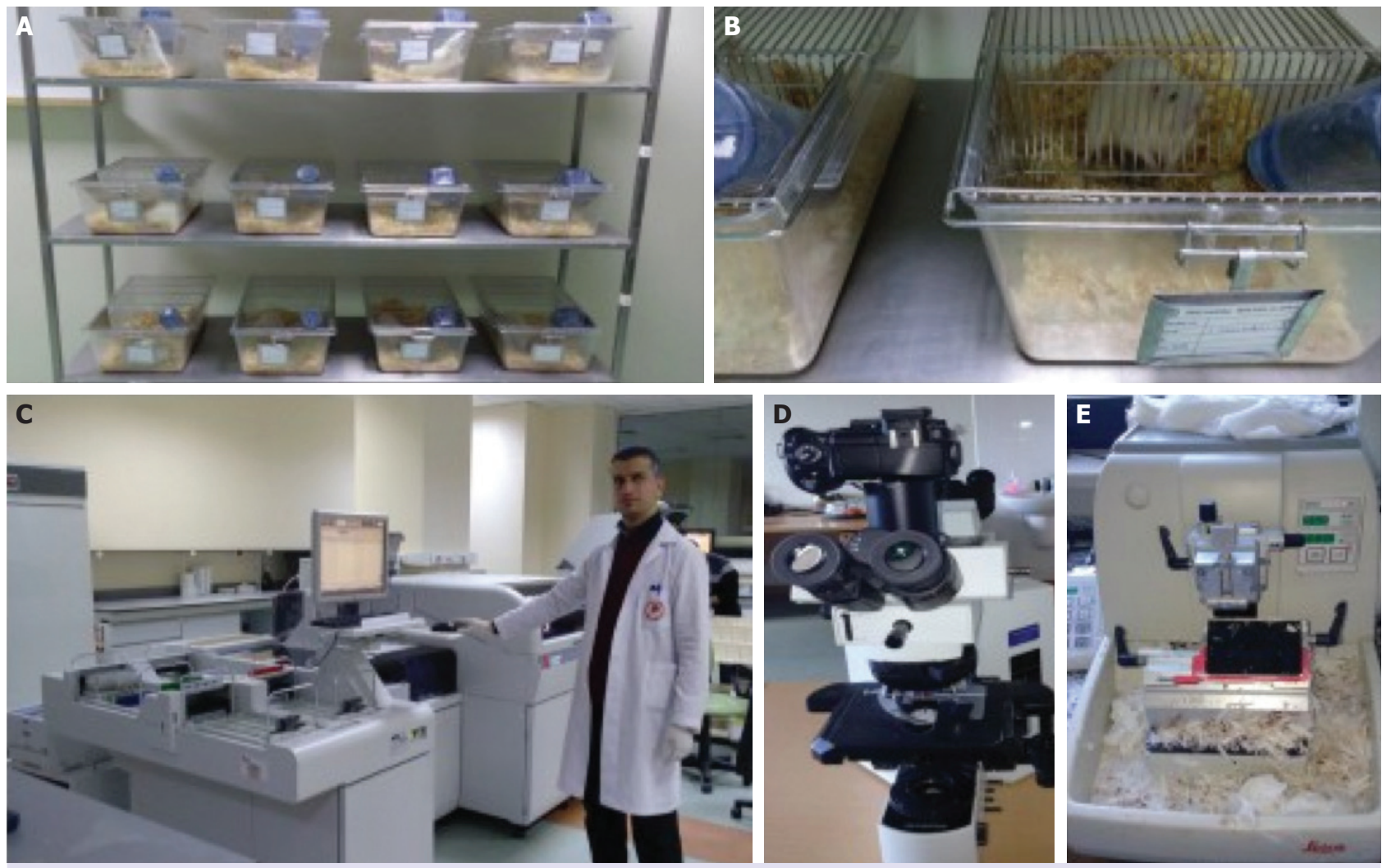

FIGURE 1. Animal experiment, total antioxidant status, total oxidant status, and pathological analyses. (A) Housing of the experimental animals. (B) Each rat was kept individually in a standard cage. (C) The auto-analyzer used for oxidantantioxidant analyses (AU5800; Beckman Coulter, Inc., Brea, CA, USA) and researcher. (D) The optical microscope and integrated camera system used in the histopathological analysis (BX51; Olympus Corp., Tokyo, Japan). (E) The microtome device used in the histopathological analysis (RM 2245; Leica Biosystems Nussloch GmbH, Wetzlar, Germany).

OSI $=$ TOS/TAS. The principle of TAS measurement is that antioxidants in the sample reduce the dark blue-green colored 2-2'-Azino-bis (3-ethylbenzthiazoline-6-sulfonic acid) (ABTS) radical to a colorless, reduced form of ABTS. The change of absorbance at $660 \mathrm{~nm}$ is related to the total antioxidant level of the sample. The assay is calibrated with a stable antioxidant standard solution known as Trolox Equivalent, which is similar to vitamin $E$ [29]. The principle of TOS measurement is that oxidants present in the sample oxidize the ferrous ion-chelator complex into ferric ion. The oxidation reaction is prolonged by enhancer molecules, which are abundantly present in the reaction medium. The ferric ion provides a colored complex with chromogen in an acidic medium. The color intensity, which can be measured spectrophotometrically, is related to the total amount of oxidant molecules present in the sample. The assay is calibrated with hydrogen peroxide [30].

\section{Histopathological examination}

Tissues used for histopathological examination were fixed in $10 \%$ neutral formalin after samples were symmetrically divided in half on a vertical axis down the middle. Following dehydration and paraffin inclusion procedure, the tissues were embedded into paraffin blocks. Slices in thickness of 4 to $5 \mu \mathrm{m}$ were obtained from the paraffin blocks using a rotary microtome (RM 2245; Leica Biosystems Nussloch GmbH, Wetzlar, Germany). The slices were stained with hematoxylin-eosin. Following the staining procedure, all of the slices were examined under an optical microscope (BX51, Olympus Corp., Tokyo, Japan), evaluated, and photographed. 
TABLE 1. The total antioxidant-oxidant status and oxidative stress index values of the tissues

\begin{tabular}{|c|c|c|c|c|}
\hline Groups & $\begin{array}{c}\mathrm{AL} \\
\text { Mean } \pm \mathrm{SD}\end{array}$ & $\begin{array}{c}\text { TM } \\
\text { Mean } \pm \text { SD }\end{array}$ & $\begin{array}{c}\text { TM-CR } \\
\text { Mean } \pm \text { SD }\end{array}$ & $\begin{array}{c}\text { Kruskal-Wallis } \\
\mathrm{p}\end{array}$ \\
\hline Serum TAS ( $\mu$ mol Trolox Eq/L) & $1.52 \pm 0.14$ & $1.74 \pm 0.31$ & $1.69 \pm 0.26$ & 0.239 \\
\hline Serum TOS $\left(\mu \mathrm{mol} \mathrm{H}_{2} \mathrm{O}_{2} \mathrm{Eq} / \mathrm{L}\right)$ & $16.03 \pm 3.08$ & $14.40 \pm 5.07$ & $14.65 \pm 5.86$ & 0.05 \\
\hline Serum OSI & $10.73 \pm 2.79$ & $8.23 \pm 2.08$ & $8.63 \pm 2.76$ & 0.294 \\
\hline Liver TAS ( $\mu \mathrm{mol}$ Trolox Eq/gr prt) & $0.2 \pm 0.02$ & $0.22 \pm 0.01 *$ & $0.21 \pm 0.01$ & 0.041 \\
\hline Liver TOS $\left(\mu \mathrm{mol} \mathrm{H}_{2} \mathrm{O}_{2} \mathrm{Eq} / \mathrm{gr} \mathrm{prt}\right)$ & $2.14 \pm 0.19$ & $2.28 \pm 0.27$ & $2.20 \pm 0.21$ & 0.344 \\
\hline Liver OSI & $10.89 \pm 1.75$ & $10.50 \pm 1.45$ & $10.39 \pm 1.32$ & 0.898 \\
\hline Striated muscle TAS ( $\mu \mathrm{mol}$ Trolox Eq/gr prt) & $0.15 \pm 0.01$ & $0.18 \pm 0.06$ & $0.18 \pm 0.02$ & 0.111 \\
\hline Striated muscle TOS ( $\mu \mathrm{mol} \mathrm{H}_{2} \mathrm{O}_{2} \mathrm{Eq} / \mathrm{gr}$ prt) & $0.34 \pm 0.08$ & $0.3 \pm 0.05$ & $0.34 \pm 0.11$ & 0.57 \\
\hline Striated muscle OSI & $2.32 \pm 0.57$ & $1.83 \pm 0.69$ & $1.89 \pm 0.45$ & 0.116 \\
\hline Adipose tissue TAS ( $\mu \mathrm{mol}$ Trolox Eq/gr prt) & $0.30 \pm 0.14$ & $0.43 \pm 0.15$ & $0.50 \pm 0.23$ & 0.122 \\
\hline Adipose tissue TOS $\left(\mu \mathrm{mol} \mathrm{H}_{2} \mathrm{O}_{2} \mathrm{Eq} / \mathrm{gr}\right.$ prt & $30.50 \pm 2.18$ & $14.38 \pm 1.35 *$ & $13.69 \pm 1.73 *$ & $<0.001$ \\
\hline Adipose tissue OSI & $12.65 \pm 6.18$ & $3.75 \pm 1.60 *$ & $3.40 \pm 1.83 *$ & 0.001 \\
\hline Brain TAS ( $\mu$ mol Trolox Eq/gr prt) & $0.23 \pm 0.02$ & $0.25 \pm 0.03$ & $0.25 \pm 0,03$ & 0.286 \\
\hline Brain TOS $\left(\mu \mathrm{mol} \mathrm{H}_{2} \mathrm{O}_{2} \mathrm{Eq} / \mathrm{gr}\right.$ prt & $2.75 \pm 0.42$ & $2.22 \pm 0.55$ & $2.40 \pm 0.51$ & 0.108 \\
\hline Brain OSI & $12.19 \pm 2.16$ & $9.33 \pm 3.29$ & $10.01 \pm 2.91$ & 0.153 \\
\hline
\end{tabular}

AL: Ad libitum; OSI: Oxidative stress index (TOS/TAS); SD: Standard deviation; TAS: Total antioxidant status; TM: Two meals; TM-CR: Two meals and calories restricted by $20 \%)$; TOS: Total oxidant status. $*$ When compared with AL group, these were statistically significant (Mann-Whitney U; $p<0.05)$.

The materials used in the animal experiment and for the histopathological and biochemical analyses and animal experiments are shown in Figure 1.

\section{Statistical analysis}

Statistical analyses were conducted using the IBM SPSS Statistics for Windows, Version 20.0 (IBM Corp., Armonk, NY, USA) program. The numerical data obtained were expressed as mean $\pm \mathrm{SD}$. Since there was a small number of subjects in each group, the Kruskal-Wallis test, which is a nonparametric test, was used to compare the average TAS, TOS, and OSI values of the 3 groups. Chi-square test was used to statistically analyze the rat liver histopathology.

\section{RESULTS}

Findings on oxidative stress

and antioxidant system

The effects of meal frequency and calorie restriction on TAS ( $\mu$ mol Trolox Eq/L), TOS $\left(\mu \mathrm{mol} \mathrm{H}_{2} \mathrm{O}_{2}\right.$ $\mathrm{Eq} / \mathrm{L}$ ), and OSI values in the serum of the rats were analyzed (mean $\pm \mathrm{SD})$. There were significant differences between the AL and TM, and the AL and TM-CR groups in the adipose tissue, and between the AL and TM groups in the livers of the rats (Table 1).

\section{Results of the histopathological}

analysis of the rat livers

At the end of the experiment, the rat livers were microscopically analyzed in terms of granularity, changes in hepatocyte array, fattening, inflammation, and fibrosis. No fattening of the liver, inflammation, or fibrosis was seen in the experimental animals. Granularity in hepatocytes and hepatocyte array disorders were noted in some liver samples (Figure 2). In the results of the analysis using the chi-square test, it was determined that the granularity and changes in hepatocyte organization were not statistically significant. No significant differences were found between groups in terms of the param- 

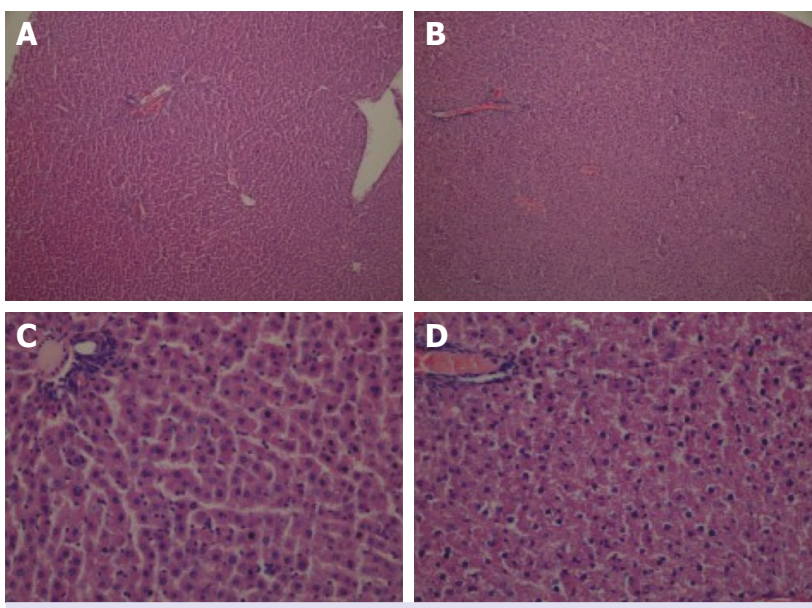

FIGURE2. Histopathology of rat liver. (A) Rat liver. Normal histology. Hepatocyte array is normal (HE x40).

(B) Mild disorder in hepatocyte array (HE x40). (C) Mild disorder in hepatocyte array and mild increase in cytoplasmic granularity. Normal (HE x200). (D) Noticeable disorder in hepatocyte array and noticeable increase in cytoplasmic granularity (HE x200).

eters analyzed. Examples of the microscopic images of the rat livers are shown in Figure 2.

\section{DISCUSSION}

The results of our study were consistent with the literature: TAS level increased significantly in the TM and TM-CR groups compared with the control group $(\mathrm{p}<0.05)$. Based on the analysis in terms of TOS and OSI, a decrease was seen in the TM and TM-CR groups in comparison with the control group. However, there was not a statistical significance, except in fat tissue. In the fat tissue, in terms of TOS and OSI levels, the TM and TM-CR groups showed significant decreases in comparison with the control group $(p<0.05)$. The relationship between manner of feeding and antioxidant activity was demonstrated with a noticeable increase in antioxidant activity, which is the protective mechanism of the body against oxidative stress on the liver, one of the central organs of metabolism.

As a result, it was seen that TM and TM-CR methods of feeding may be useful in reducing oxidative stress and increasing antioxidant activity, as previously demonstrated in the literature. In our study, the TM-CR group was allowed to feed 2 times a day and subjected to a moderate level of calorie restriction (20\%). It was determined that the TAS level in the liver of the TM group increased significantly in comparison to the AL group, while TOS and OSI levels in the fat tissue decreased significantly in the TM and TM-CR groups. Considering the oxidantantioxidant system results of the TM-CR group according to the literature, many studies have reported a reduction of oxidative activity with calorie restriction, which is in agreement with the results of our study [21-27]. However, there are a limited number of studies reporting an increase in antioxidant activity with calorie restriction $[26,27]$. The fact that not all studies implementing calorie restriction demonstrated an increase in antioxidant activity has been tied to the duration of implementation [27]. Increase in antioxidant activity with calorie restriction was observed in our study, which may be considered long-term in comparison to other reported studies [21-27]. Moreover, the combined implementation of meal frequency and calorie restriction, and positive outcomes regarding the antioxidant system, increases the value of our study. Another important result of this study is that meal frequency implementation without calorie restriction, which has not been sufficiently investigated, was shown to have positive effects on the oxidant-antioxidant system.

No harm to the liver was seen in the analysis of liver histopathology with respect to meal frequency or calorie restriction. The granulation and change in the hepatocyte array observed more in the TM and TM-CR groups was insignificant, and may be explained by increased metabolic activity and the increase in the number of organelles, such as granulated endoplasmic reticulum, that have an active role in metabolism.

This study produced promising findings about the relationship between meal frequency and the antioxidant system, considering that there is a limited amount of data in the literature on this subject. The results of our study indicated that, like calorie restriction, reducing meal frequency probably strengthens the antioxidant system and reduces oxidative stress. Therefore, eating less and eating less frequently may be presented as an alternate protec- 
tive treatment in the prevention of hundreds of diseases known to be related to oxidative stress [2830]. The amount and frequency of food intake are as important as the content. Future studies of longer duration, and even life-long observation studies regarding the effects on life span, the addition of the factor of sex, and groups combining nutrition and exercise, may lead to new findings and may confirm our results. Changes in the TAS, TOS, and OSI values in the TM and TM-CR groups were in the same direction as the AL group; the fact that most of these changes were statistically insignificant may be explained by the small number of subjects. Statistical significance might arise in studies with a larger number of subjects.

Conflict of Interest: No conflict of interest was declared by the authors.

Financial Disclosure: The study was supported by the Scientific Research Unit (SDÜ BAP) and Faculty Development Program (OYP Coordination Unit; SDÜ ÖYP) of Suleyman Demirel University (Project Numbers: SDÜ BAP 4476-ÖYP-D2-15) and ÖYP (ÖYP 05333-DR-12) unit).

Authorship contributions: Concept - H.B.S., F.G.; Design H.B.S., F.G.; Supervision - H.B.S., F.G.; Materials - H.B.S., F.G., I.M.C.; Data collection \&/or processing - H.B.S., F.G., I.M.C.; Analysis and/or interpretation - H.B.S., F.G.; Literature search - H.B.S., F.G.; Writing - H.B.S., F.G., I.M.C.; Critical review H.B.S., F.G.

\section{REFERENCES}

1. Gürdöl F. Bilimin Mum Işığında Yemek; Beslenmenin Biyokimyası. Nobel Tip Kitabevleri; 2014.

2. Steffens AB. Blood glucose and FFA levels in relation to the meal pattern in the normal rat and the ventromedial hypothalamic lesioned rat. Physiol Behavior 1969;4:215-6.

3. Lima FB, Hell NS, Timo-Iaria C. Carbohydrate metabolism and food intake in food-restricted rats. Relationship between the metabolic events during the meal and the degree of food intake. Physiol Behav 1985;35:695-700.

4. Glendinning JI, Smith JC. Consistency of meal patterns in laboratory rats. Physiol Behav 1994;56:7-16.

5. Surina-Baumgartner DM, Arnold M, Moses A, Langhans W. Metabolic effects of a fat- and carbohydrate-rich meal in rats. Physiol Behav 1996;59:973-81.

6. Melhorn SJ, Krause EG, Scott KA, Mooney MR, Johnson JD, Woods SC, et al. Acute exposure to a high-fat diet alters meal patterns and body composition. Physiol Behav 2010;99:33-9.

7. Romsos DR, Leveille GA. Effect of meal frequency and diet com- position on glucose tolerance in the rat. J Nutr 1974;104:150312 .

8. Wolever TM. Metabolic effects of continuous feeding. Metabolism 1990;39:947-51.

9. Stote KS, Baer DJ, Spears K, Paul DR, Harris GK, Rumpler WV, et al. A controlled trial of reduced meal frequency without caloric restriction in healthy, normal-weight, middle-aged adults. Am J Clin Nutr 2007;85:981-8.

10. Muiruri KL, Leveille GA. Metabolic adaptations in meal-fed rats: effects of increased meal frequency or ad libitum feeding in rats previously adapted to a single daily meal. J Nutr 1970;100:450-60.

11. Ohkawara K, Cornier MA, Kohrt WM, Melanson EL. Effects of increased meal frequency on fat oxidation and perceived hunger. Obesity (Silver Spring) 2013;21:336-43.

12. Mattson MP. Energy intake, meal frequency, and health: a neurobiological perspective. Annu Rev Nutr 2005;25:237-60.

13. Koopman KE, Caan MW, Nederveen AJ, Pels A, Ackermans MT, Fliers E, et al. Hypercaloric diets with increased meal frequency, but not meal size, increase intrahepatic triglycerides: a randomized controlled trial. Hepatology 2014;60:545-53.

14. Hursting SD, Lavigne JA, Berrigan D, Perkins SN, Barrett JC. Calorie restriction, aging, and cancer prevention: mechanisms of action and applicability to humans. Annu Rev Med 2003;54:131-52.

15. Cantó C, Auwerx J. Caloric restriction, SIRT1 and longevity. Trends Endocrinol Metab 2009;20:325-31.

16. Prolla TA, Mattson MP. Molecular mechanisms of brain aging and neurodegenerative disorders: lessons from dietary restriction. Trends Neurosci 2001;24:S21-31.

17. Mattson MP, Chan SL, Duan W. Modification of brain aging and neurodegenerative disorders by genes, diet, and behavior. Physiol Rev 2002;82:637-72.

18. Bruce-Keller AJ, Umberger G, McFall R, Mattson MP. Food restriction reduces brain damage and improves behavioral outcome following excitotoxic and metabolic insults. Ann Neurol 1999;45:8-15.

19. Contestabile A, Ciani E, Sparapani M, Guarnieri T, Dell'Erba $\mathrm{G}$, Bologna $\mathrm{F}$, et al. Activation of the ornithine decarboxylasepolyamine system and induction of $\mathrm{c}$-fos and $\mathrm{p} 53$ expression in relation to excitotoxic neuronal apoptosis in normal and microencephalic rats. Exp Brain Res 1998;120:519-26.

20. Drew B, Phaneuf S, Dirks A, Selman C, Gredilla R, Lezza A, et al. Effects of aging and caloric restriction on mitochondrial energy production in gastrocnemius muscle and heart. Am J Physiol Regul Integr Comp Physiol 2003;284:R474-80.

21. Dubnov G, Kohen R, Berry EM. Diet restriction in mice causes differential tissue responses in total reducing power and antioxidant compounds. Eur J Nutr 2000;39:18-30.

22. Xia E, Rao G, Van Remmen H, Heydari AR, Richardson A. Activities of antioxidant enzymes in various tissues of male Fischer 344 rats are altered by food restriction. J Nutr 1995;125:195201. 
23. Leeuwenburgh C, Wagner P, Holloszy JO, Sohal RS, Heinecke JW. Caloric restriction attenuates dityrosine cross-linking of cardiac and skeletal muscle proteins in aging mice. Arch Biochem Biophys 1997;346:74-80.

24. Dixit R, Coleman JB, Mattson B, Balam GC, Kenan KP, St. Louis MO. The effects of uncontrolled excess caloric intake and moderate to marked caloric restriction (CR) on obesity, spontaneous disease and cancers in sprague dowley (SD) rats. Toxicology Letters 1998;95:182.

25. Dirks AJ, Leeuwenburgh C. Caloric restriction in humans: potential pitfalls and health concerns. Mech Ageing Dev 2006; $127: 1-7$.

26. Louala S, Benyahia-Mostefaoui A, Lamri-Senhadji MY. Energy restriction reduces oxidative stress in the aorta and heart and corrects the atherogenic risk in obese rat. Ann Cardiol Angeiol (Paris) 2013;62:155-60.

27. Stankovic M, Mladenovic D, Ninkovic M, Vucevic D, Tomasevic $\mathrm{T}$, Radosavljevic T. Effects of caloric restriction on oxidative stress parameters. Gen Physiol Biophys 2013;32:277-83.

28. Halliwell B, Gutteridge JMC. Free radicals in biology and medicine. $3^{\text {rd }}$ ed. Oxford: Oxford Science Publications; 2000.

29. Erel O. A novel automated direct measurement method for total antioxidant capacity using a new generation, more stable ABTS radical cation. Clin Biochem 2004;37:277-85.

30. Erel O. A new automated colorimetric method for measuring total oxidant status. Clin Biochem 2005;38:1103-11. 